\title{
ON A CLASS OF FOLIATIONS AND THE EVALUATION OF THEIR CHARACTERISTIC CLASSES
}

\author{
BY DANIEL BAKER
}

Communicated by J. A. Wolf, January 3, 1977

This note discusses a class of foliations and a technique for evaluating the generalized Godbillon-Vey invariants on these foliations. The information obtained yields information about the cohomology of the Haefliger spaces $H^{*}\left(B \Gamma_{n}^{r}, \mathbf{R}\right)$ and $H^{*}\left(F \Gamma_{n}^{r}, \mathbf{R}\right), r \geqslant 2$. The class of foliations contains examples which have been studied by others as well. In particular, the foliations examined in [KT2] and in [Y] are of this type.

Let $G^{\mathbf{C}}$ be a complex semisimple Lie group. There is a class of subgroups of $G^{\mathbf{C}}$ called parabolic subgroups, and the conjugacy classes of these subgroups are in 1-1 correspondence with subsets of the Dynkin diagram for $\widehat{G}^{\mathbf{C}}$, the Lie algebra of $G^{\mathrm{C}}$ (see [S] for a more detailed exposition). If $P^{\mathrm{C}}$ is a parabolic subgroup then the Lie algebra $P^{\mathbf{C}}$ of $P^{\mathbf{C}}$ can be written in the form $P^{\mathbf{C}}=G_{1}^{\mathbf{C}} \oplus$ $T_{1}^{\mathbf{C}} \oplus N^{\mathbf{C}}$. Here $G_{1}^{\mathbf{C}}$ is semisimple and has a Dynkin diagram obtained by removing the subset of vertices mentioned above from the Dynkin diagram for $G^{\mathbf{C}}$. $T_{1}^{\mathbf{C}}$ is an abelian subalgebra of $G^{\mathbf{C}}, G_{1}^{\mathbf{C}} \oplus T_{1}^{\mathbf{C}}$ contains a Cartan subalgebra of $G^{\mathbf{C}}$, and $N^{\mathbf{C}}$ is a nilpotent subalgebra. In fact, $G^{\mathbf{C}}=G_{1}^{\mathbf{C}} \oplus T_{1}^{\mathbf{C}} \oplus N^{\mathbf{C}} \oplus N^{-\mathbf{C}}$ where $N^{-\mathbf{C}}$ is a nilpotent subalgebra isomorphic to $N^{\mathbf{C}}$, and $\left[G_{1}^{\mathbf{C}}, T_{1}^{\mathrm{C}}\right]=0,\left[G_{1}^{\mathbf{C}} \oplus T_{1}^{\mathrm{C}}, N^{\mathbf{C}}\right]$ $\subset N^{\mathbf{C}},\left[G_{1}^{\mathbf{C}} \oplus T_{1}^{\mathbf{C}}, N^{-\mathbf{C}}\right] \subset N^{-\mathbf{C}}$.

Now let $G$ be a real form of $G^{C}$ such that $G=G_{1} \oplus T_{1} \oplus N \oplus N^{-}$where $G_{1}=G_{1}^{C} \cap G$, etc. Then $G$ has a subalgebra $P=G_{1} \oplus T_{1} \oplus N$. If $G$ has Lie algebra $G$, then there is a discrete subgroup, $\Gamma \subset G$, with $\Gamma G$ a compact manifold (see [R]), and the left translates of $P$ determine a foliation on $\Gamma \mid G$. This is the foliation we study.

Let $W_{n}=P_{n}\left[c_{1}, \ldots, c_{n}\right] \otimes \Lambda^{*}\left(u_{1}, \ldots, u_{n}\right)$ be the cochain complex with $\operatorname{deg} c_{i}=2 i, \operatorname{deg} u_{i}=2 i-1, d c_{i}=0, d u_{i}=c_{i} . P_{n}\left[c_{1}, \ldots, c_{n}\right]$ is the polynomial algebra in $c_{1}, \ldots, c_{n}$, truncated above deg $2 n$ where $n$ is the codimension of the above foliation. There is a map $\varphi: H^{*}\left(W_{n}, \mathbf{R}\right) \rightarrow H^{*}(\Gamma \backslash G, \mathbf{R})$ giving characteristic classes for the foliation (see [BT] for the construction of $\varphi$ ). We analyse this map $\varphi$.

First note that, since a left invariant form on $G$ induces a form in $\Lambda^{*}(\Gamma \backslash G, \mathbf{R})$, there is a map $\alpha: H^{*}(G, \mathbf{R}) \rightarrow H^{*}(\Gamma \backslash G, \mathbf{R})$ where $H^{*}(G, \mathbf{R})$ is the cohomology of the Lie algebra $G$.

$A M S$ (MOS) subject classifications (1970). Primary 57D20, 57D30. 
Proposition (SEe Also Lemma 4.88 [KT1]). There is a commutative diagram where the map $\alpha$ is injective.

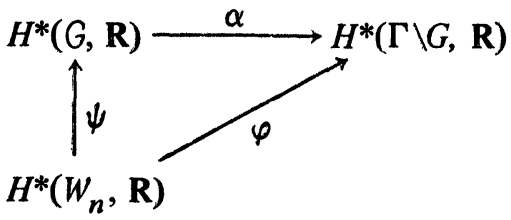

We analyze the map $\psi$. Let $\bar{G}$ be a compact form of $G$. The key observation is that $H^{*}(G, \mathrm{C}) \approx H^{*}(\bar{G}, \mathrm{C})$. Let $\bar{G}_{1}, \bar{T}_{1}$ be the subgroups of $\bar{G}$ corresponding to $G_{1}^{\mathrm{C}}, T_{1}^{\mathrm{C}}$. We use the results of [BO] to describe $H^{*}(\bar{G}, \mathbf{C})$ in terms of the spectral sequence for the bundle $\bar{G}_{1} \times \bar{T}_{1} \rightarrow \bar{G} \rightarrow \bar{G} / \bar{G}_{1} \times \bar{T}_{1}$. Specifically, let $x_{1}, \ldots, x_{n} \in H^{*}\left(\bar{G}_{1} \times \bar{T}_{1}, \mathrm{C}\right)$ be the primitive elements transgressing to $g_{1}, \ldots$, $g_{n} \in H^{*}\left(B_{\bar{G}_{1} \times \bar{T}_{1}}, \mathrm{C}\right)=S$. Let $\rho: H^{*}\left(B_{\bar{G}}, \mathrm{C}\right) \rightarrow S$ represent the map on characteristic classes induced by inclusion $\bar{G}_{1} \times \bar{T}_{1} \subset \bar{G}$. Let $I \subset S$ be the ideal generated by the image of $\rho$. Let $A=S / I \otimes H^{*}\left(\bar{G}_{1} \times \bar{T}_{1}\right.$, C) be the complex with $d\left(1 \otimes x_{i}\right)=g_{i} \otimes 1, d\left(g_{i} \otimes 1\right)=0$. Then $H^{*}(A, \mathbf{C}) \approx H^{*}(\bar{G}, \mathbf{C}) \approx H^{*}(G, \mathbf{C})$.

There is a homomorphism $\sigma: \bar{G}_{1} \times \bar{T}_{1} \rightarrow \operatorname{Gl}(n, \mathrm{C})$ given by the adjoint representation of $\bar{G}_{1} \times \bar{T}_{1}$ on the Lie algebra $N^{-C} . \sigma$ induces a map $\overline{\boldsymbol{\sigma}}: H^{*}\left(B_{\mathrm{Gl}(n, \mathrm{C})}, \mathrm{C}\right) \rightarrow S$. For each Chern class $c_{k}$ we can choose an element $\xi_{k}$ in the acyclic complex $S \otimes H^{*}\left(\bar{G}_{1} \times \bar{T}_{1}, \mathrm{C}\right), d\left(g_{i} \otimes 1\right)=0, d\left(1 \otimes x_{i}\right)=g_{i} \otimes 1$, with $d \xi_{k}=\bar{\sigma}\left(c_{k}\right) \otimes 1 . \xi_{k}$ determines an element $\bar{\xi}_{k}$ in $A$. Then we have a map $\nu: W_{n} \rightarrow A, \nu\left(c_{k}\right)=(\sqrt{-1})^{k} \bar{\sigma}\left(c_{k}\right) \otimes 1, \nu\left(u_{k}\right)=(\sqrt{-1})^{k} \bar{\xi}_{k}$.

THEOREM. There is a commutative diagram where $\gamma$ is induced by the coefficient map $\mathbf{R} \subset \mathbf{C}$, and $\bar{\alpha}$ is injective

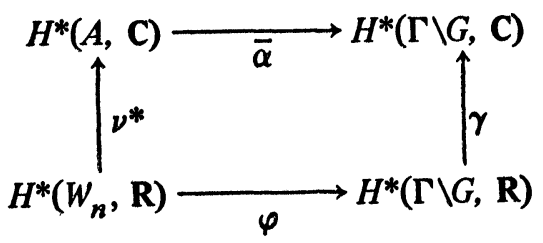

The power of this theorem stems from the fact that $A$ is a finitely generated complex whose cohomology is an exterior algebra on the primitive elements of $\bar{G}$. Thus, given a cocycle in $A$, it is feasible to try to determine the class it lies in.

EXAMPLES. Let $G=\operatorname{sl}(n+k, \mathbf{R}), k<n$ or $k=n=1$,

$$
\begin{aligned}
G_{1} \oplus T_{1} \approx \operatorname{sl}(n, \mathbf{R}) \oplus \mathrm{sl}(k, \mathbf{R}) \oplus \mathbf{R} \\
=\left\{\left\|a_{i j}\right\| \in \mathrm{sl}(n+k, \mathbf{R}) \mid a_{i j}=0 \text { for } i>k, j \leqslant k \text { or } i \leqslant k, j>k\right\}, \\
\quad P=\left\{\left\|a_{i j}\right\| \in \mathrm{sl}(n+k, \mathbf{R}) \mid a_{i j}=0 \text { for } i>k, j \leqslant k\right\} .
\end{aligned}
$$

Then in $H^{*}(\Gamma \operatorname{SL}(n+k, \mathbf{R}), \mathbf{R})$ (and thus in $H^{*}\left(F \Gamma_{n k}^{r}, \mathbf{R}\right)$ ) the classes $c_{1}^{n k} u_{1} \cdots u_{k} u_{i_{1}} \cdots u_{i_{l}}$ for all $k<i_{1}<\cdots<i_{k} \leqslant n$ are nonzero and linearly 
independent (this includes the class $c_{1}^{n k} u_{1} \cdots u_{k}$ ).

These results have been obtained by Kamber and Tondeur for the case when $k=1$ and can be found in [KT1] and [KT2].

It is possible to obtain information about the independence of classes when $c_{1}^{n k}$ is replaced by another monomial in $c_{1}, \ldots, c_{n k}$ by comparing examples for different values of $n$ and $k$. For instance, by comparing the example $k=2, n=$ $q$ with the example $k=1, n=2 q$ one obtains: For $q \neq 2$, in $H^{*}\left(F \Gamma_{2 q}^{r}, \mathbf{R}\right)$ the set of classes

$$
\left\{c_{1}^{2 q} u_{1} u_{2} u_{i_{1}} \cdots u_{i_{l}}, c_{2} c_{1}^{2 q-2} u_{1} u_{2} u_{i_{1}} \cdots u_{i_{l}} \mid 2<i_{1}<\cdots<i_{l} \leqslant q\right\}
$$

are linearly independent.

By examining foliations on $\Gamma G / K$, where $K$ is a compact subgroup of $P$, analogous information for classes in $H^{*}\left(B \Gamma_{n}^{r}, \mathrm{R}\right)$ is obtained. For example, in $H^{*}\left(B \Gamma_{2 q}^{r}, \mathbf{R}\right)$ the set of classes

$$
\begin{aligned}
\left\{c_{1}^{2 q} u_{1} u_{i_{1}} \cdots u_{i_{l}}, c_{2} c_{1}^{2 q-2} u_{1} u_{i_{1}} \cdots u_{i_{l}} \mid\right. \\
\left.1<i_{1}<\cdots<i_{l} \leqslant q \text { and the } i_{j} \text { are odd }\right\}
\end{aligned}
$$

are linearly independent.

For these constructions, other examples, and a more detailed exposition, see $[\mathrm{B}]$.

\section{REFERENCES}

[B] D. Baker, On a class of foliations and the evaluation of their characteristic classes, Ph. D. Thesis, SUNY, Stony Brook, 1976.

[BO] A. Borel, Sur la cohomologie des espaces fibrés princtpaux et des espaces homogènes de groupes de Lie compacts, Ann. of Math. (2) 57 (1953), 115-207. MR 14, 490.

[BT] R. Bott, Lectures on characteristic classes and foliations, (Notes by L. Conlon, Mexico, 1971), Lecture Notes in Math., vol. 279, Springer-Verlag, Berlin and New York, 1972. MR 50 \#14777.

[KT1] F. Kamber and Ph. Tondeur, Foliated bundles and characteristic classes, Lecture Notes in Math., vol. 493, Springer-Verlag, Berlin and New York, 1975. (to appear).

[KT2] - On the linear independence of certain cohomology classes of $B \Gamma$

[R] M. S. Raghunathan, Discrete subgroups of Lie groups, Ergebnisse, Band 68, Springer-Verlag, Berlin and New York, 1972.

[S] I. Satake, Classification theory of semi-simple algebraic groups, Marcel Dekker, New York, 1971. MR 47 \#5135.

[Y] K. Yamato, Examples of foliations with non trivial characteristic classes, Osaka J. Math. 12 (1975), 401-417.

DEPARTMENT OF MATHEMATICS, UNIVERSITY OF NORTH CAROLINA, CHAPEL HILL, NORTH CAROLINA 27514 